\title{
Spectrométrie à très haute résolution de l'émission $K$ du néon
}

\author{
T. Wahi, M. Bonnefoy, M. Chassevent, A. Fleury, J.J. Bonnet, X. Husson* et C. Hague**
}

Laboratoire de Physique Atomique Appliquée à l'Instrumentation X-UV, Conservatoire National des Arts et Métiers, 292 rue Saint Martin, 75141 Paris cedex 03, France

* Laboratoire de Spectrométrie Atomique, 6 boulevard du Maréchal Juin, 14050 Caen, France

** Laboratoire de Chimie Physique, Université Pierre et Marie Curie, 11 rue Pierre et Marie

Curie, 75231 Paris cedex 05, France

\begin{abstract}
A high-resolution electron-excited $\mathrm{K}$ emission spectrum has been performed. For the analysis of the radiation an X-ray spectrometer based on the Johann bent-crystal was used. The spectrum shows essentially transitions in 1-2 times ionised Neon. The $\mathrm{KL}_{1}$ and $\mathrm{KL}_{2,3}$ satellites are resolved and their intensities yield information about the formation of the multiple vacancy states. An other interest of this experiment is the high wavelength resolution obtained. This spectrometer equipped with other diffracting crystals is destinated to the study of the X-Ray spectra arising from collision of multicharged ions on metallic surfaces.
\end{abstract}

Le spectre d'émission $K$ du Néon excité par des électrons a été très étudié depuis de nombreuses années à l'aide de spectromètres à cristaux plans [1] et à réseaux [2].

Avec un spectromètre à cristal courbe conçu par C.Hague [3], il a été possible d'obtenir une résolution $\lambda / \Delta \lambda=1500$ à $\lambda=1.461 \mathrm{~nm}$. Dans une enceinte à vide $\left(10^{-6} \mathrm{hPa}\right)$, sont disposés:

* une chambre de collision escamotable

* un cristal de Béryl courbé $(2 \mathrm{~d}=1.595 \mathrm{~nm})$ de rayon de courbure égale à $500 \mathrm{~mm}$

* un compteur proportionnel à flux gazeux.

La cible gazeuse de Néon à la pression de $2.10^{-2} \mathrm{hPa}$ est excitée par un faisceau d'électrons $(2.8 \mathrm{keV}, 0.5 \mathrm{~mA})$.

Une fente (réglable) de $100 \mu \mathrm{m}$ d'ouverture permet de maintenir la forte différence de pression entre chambre cible et enceinte à vide, et d'améliorer la résolution. Ce dispositif équipé d'autres réflecteurs (multicouches) est destiné à des études de transitions $\mathrm{X}$ à plus grandes longueurs d'onde émises lors de l'interaction entre ions multichargés lents et une surface métallique.

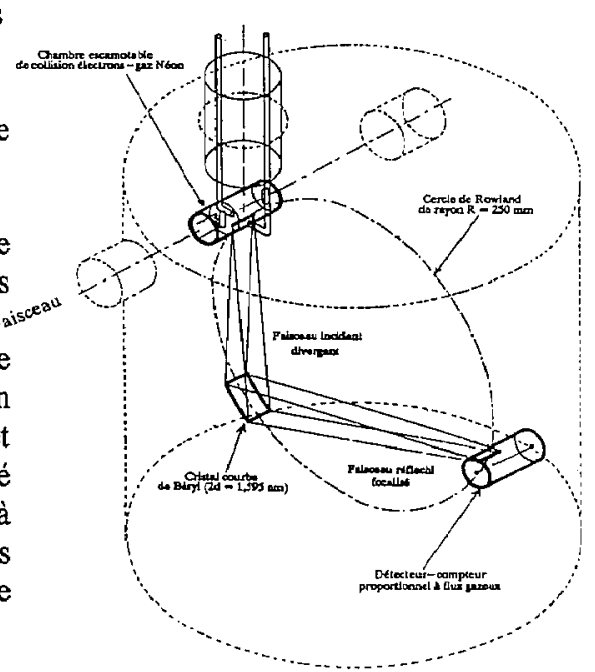

Fig.1: Schéma du montage expérimental 

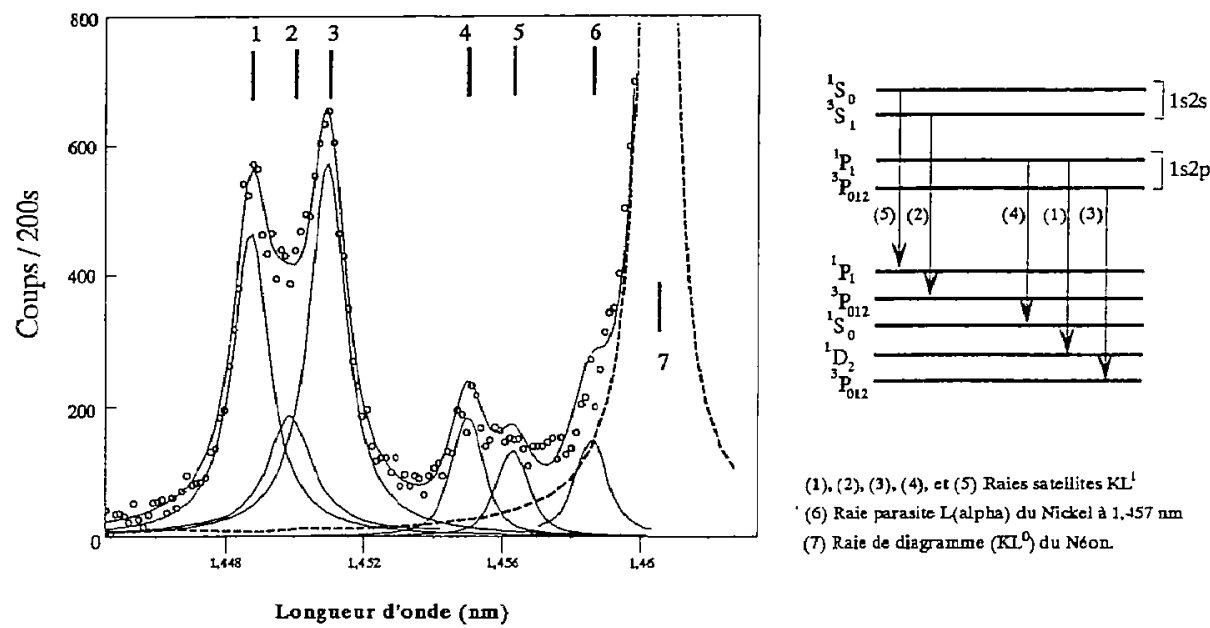

(1). (2), (3). (4), et (5) Raies satellites KL

(6) Raie parasite L(alpha) du Nickel à 1,457 $\mathrm{nm}$

(T) Raie de diagrame (KI 9 du Nón

Fig. 2: Spectre montrant les raies issues des différents multiplets (diagramme à droite) de la configuration initiale $\mathrm{KL}^{1}\left(\mathrm{Ne}^{2+}\right)$.

Comme l'indique la fig.2, la résolution atteinte permet pour la première fois en spectrométrie à analyseur focalisant, une étude détaillée des émissions satellites qui apparaissent sur la partie courte longueur d'onde du spectre par rapport à l'émission de diagramme $K \alpha(7)$. Ces émissions satellites $(1,2,3,4$ et 5 sur le diagramme des transitions ci-dessus à droite) sont issues des différentes transitions multiplets dans le Néon doublement ionisé $\mathrm{Ne}^{2+}\left(\mathrm{KL}^{\mathbf{l}}\right)$.

L'évaluation des intensités relatives de ces transitions multiplets et l'utilisation des rendements de fluorescence théoriques [4] correspondants à chacun des multiplets, permet d'accéder aux rapports de probabilités des populations multiplets dans les différentes configurations initiales $1 \mathrm{~s} 2 \mathrm{~s}$ et $1 \mathrm{~s} 2 \mathrm{p}$ (Tableau ci-dessous). Les résultats obtenus, en comparaison avec ceux d'Ågren et al [2] et Chattarji et al [5], confirment le caractère non statistique des populations multiplets ${ }^{1} \mathrm{P}$ et ${ }^{3} \mathrm{P}$ dans l'état initial is $2 \mathrm{p}$.

\begin{tabular}{|c|c|c|c|c|}
\hline & Présent travail & Agren ef al & $\begin{array}{c}\text { Rapports } \\
\text { théariques }\end{array}$ & \\
\hline $\begin{array}{l}{ }^{1} \mathbf{S} /{ }^{3} \mathbf{S} \\
\text { Dans la configuration } \\
\text { initial is } 2 \mathrm{~s}\end{array}$ & 0.38 & 0.31 & $\begin{array}{r}\text { Hypothese de } \\
\text { la Population } \\
0.31\end{array}$ & Statistique \\
\hline $\begin{array}{l}{ }^{1} \mathbf{P} /{ }^{3} \mathbf{P} \\
\text { Dans la configuration } \\
\text { initial } 1 \mathrm{~s} 2 \mathrm{p}\end{array}$ & 0.5 & 0.56 & 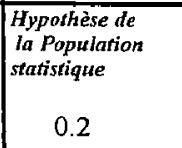 & 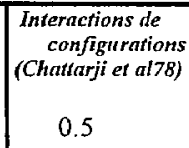 \\
\hline
\end{tabular}

[1] R.E.Lavilla, Phys. Rev. A,4,2 (1971) 476-480

[2] H Ågren et al, J. Electron Spectro. Relat.Phenom. 14 (1978) 27-39

[3] C. Hague et al, Rev. Sci. Instrum 51 (5) (1980) 621-624

[4] C.P.Bhalla, Phys. Rev. A 12 (1975) 122

[5] D.Chattarji et al, J Electron Spectro. Relat.Phenom. 13 (1978) 